Stephanie E Weissinger ${ }^{1}$ and Jochen K Lennerz ${ }^{1}$ Institute of Pathology, University Ulm, Ulm,

Germany

E-mail: jochen.lennerz@uni-ulm.de

\section{References}

1 Parsons TM. Comment on 'A diagnostic algorithm to distinguish desmoplastic from spindle cell melanoma'. Mod Pathol 2014 (this issue).

2 Weissinger SE, Keil P, Silvers DN, et al. A diagnostic algorithm to distinguish desmoplastic from spindle cell melanoma. Mod Pathol 2014;27:524-534.

3 Orosz Z. Melan-A/Mart-1 expression in various melanocytic lesions and in non-melanocytic soft tissue tumours. Histopathology 1999;34:517-525.

4 Riccioni L, Di Tommaso L, Collina G. Actin-rich desmoplastic malignant melanoma: report of three cases. Am J Dermatopathol 1999;21:537-541.

5 Clarkson KS, Sturdgess IC, Molyneux AJ. The usefulness of tyrosinase in the immunohistochemical assessment of melanocytic lesions: a comparison of the novel T311 antibody (anti-tyrosinase) with S-100, HMB45, and A103 (anti-melan-A). J Clin Pathol 2001;54:196-200.
6 Granter SR, Weilbaecher KN, Quigley C, et al. Microphthalmia transcription factor: not a sensitive or specific marker for the diagnosis of desmoplastic melanoma and spindle cell (non-desmoplastic) melanoma. Am J Dermatopathol 2001;23:185-189.

7 Miettinen M, Fernandez M, Franssila K, et al. Microphthalmia transcription factor in the immunohistochemical diagnosis of metastatic melanoma: comparison with four other melanoma markers. Am J Surg Pathol 2001;25:205-211.

8 Prasad ML, Jungbluth AA, Iversen K, et al. Expression of melanocytic differentiation markers in malignant melanomas of the oral and sinonasal mucosa. Am J Surg Pathol 2001;25:782-787.

9 Winnepenninckx V, De Vos R, Stas $\mathrm{M}$, et al. New phenotypical and ultrastructural findings in spindle cell (desmoplastic/neurotropic) melanoma. Appl Immunohistochem Mol Morphol 2003;11: 319-325.

10 Kucher C, Zhang PJ, Pasha $\mathrm{T}$, et al. Expression of Melan-A and Ki-67 in desmoplastic melanoma and desmoplastic nevi. Am J Dermatopathol 2004;26:452-457.

11 Busam KJ, Zhao H, Coit DG, et al. Distinction of desmoplastic melanoma from non-desmoplastic melanoma by gene expression profiling. J Invest Dermatol 2005;124:412-418.

\title{
Comment on 'Testing for ALK rearrangement in lung adenocarcinoma: a multicenter comparison of immunohistochemistry and fluorescent in situ hybridization'
}

\author{
Modern Pathology (2014) 27, 1423-1424; doi:10.1038/modpathol.2014.56
}

To the Editor: I read with great interest the recent study performed by Selinger et al. ${ }^{1}$ exploring the potential utility of immunohistochemical screening for ALK rearrangements. Although the results of the study are convincingly portrayed, I did note some potential omissions worth pointing out, especially given the major impact that the study might have on the logistical approach that many laboratories might choose to take as part of the very important work-up of non-small-cell lung cancers (NSCLCs).

Although the methods outline the origin of the sample, the authors do not elaborate upon any form of $a$ priori sample size calculations. These calculations can be easily performed using web-based tools; alternatively, a number of medically oriented reviews are available explaining the calculations involved. Using the worked examples of Jones et al., ${ }^{2}$ given the assumption of ALK rearrangement in $3-4 \%$ of cases of NSCLC, ${ }^{1}$ and presuming test sensitivity of at least $95 \%$ (which is often assumed in sample size calculations for studies of screening tests $^{2}$ ), I calculated a minimum sample size of 2086. This suggested that sample size is much larger than that used by Selinger et al. ${ }^{1}$ and relates to the very low prevalence of ALK rearrangements in NSCLC.

Another vitally important omission is the complete lack of any statements relating to the statistical quantification of uncertainty (eg, 95\% confidence intervals); this violates one of the major tenets of diagnostic testing research, as set out by the STARD group. ${ }^{3}$ Admittedly, the lack of any false-negative immunohistochemical results in this study makes accurate calculation of confidence intervals difficult. Nevertheless, estimates are possible: if one uses the online tool provided by the KT-clearinghouse group $^{4}$ (with the caveat that a 0 false-negative value be estimated by a small non-zero value, eg, 0.01), the sensitivity in the current study can be estimated at $100 \%$ with $95 \%$ confidence interval ranging from as low as 64 to $100 \%$. This wide confidence interval also likely relates to undersampling.

A final comment highlighting the importance of follow-up FISH studies pursuant to a positive ALK immunohistochemical result is warranted. Such a statement is an important one in order to ensure that laboratories hoping to optimize their efficiencies not 
assume that a positive ALK immunohistochemical test equates to a positive ALK rearrangement (especially in light of the very low positive predictive value noted in this study).

\section{Disclosure/conflict of interest}

The author declares no conflict of interest.

$$
\begin{array}{r}
\text { Etienne Mahe }^{1} \\
\text { Calgary Lab Services, University of Calgary, } \\
\text { Calgary, Alberta, Canada } \\
\text { E-mail: etienne.mahe@medportal.ca }
\end{array}
$$

\section{References}

1 Selinger CI, Rogers TM, Russell PA, et al. Testing for ALK rearrangement in lung adenocarcinoma: a multicenter comparison of immunohistochemistry and fluorescent in situ hybridization. Mod Pathol 2013;26:1545-1553.

2 Jones SR, Carley S, Harrison M. An introduction to power and sample size estimation. Emerg Med J 2003;20:453-458.

3 Altman DG, Barton S, Begg CB, et al. STARD Statement: STAndards for the Reporting of Diagnostic accuracy studies. http://www.stard-statement.org/. Accessed 31 December 20132013.

4 Khandwala FT, Newton K, Wong D, et al. Statistics Calculator. 2004; http://ktclearinghouse.ca/cebm/toolbox /statscalc. Accessed 31 December 20132013.

\section{Response to Mahe}

Modern Pathology (2014) 27, 1424-1425; doi:10.1038/modpathol.2014.58

To the Editor: We thank Dr Mahe for her interest in our article and appreciate her methodological observations and thoughts on the optimal strategy for detecting $A L K$ rearrangements in non-small-cell lung cancer (NSCLC). We acknowledge that while our study describing results of ALK IHC and FISH in a cohort of 594 NSCLC patients ${ }^{1}$ is one of the largest studies reporting on ALK IHC, no single study on ALK IHC published to date has come close to the suggested minimum sample size of 2086 indicated by Mahe. ${ }^{2}$ The single largest published study on ALK IHC examined only the 5A4 antibody in 640 patients. ${ }^{3}$ Our study is the larger of only two studies that have undertaken a direct comparison of the three most commonly used commercially available antibodies and FISH, with the only other study of this kind examining 377 cases. ${ }^{4}$ The findings of our study together with the results of these and other studies have recently been compiled in the 'IASLC atlas of ALK testing in lung Cancer'5 and are consistent in demonstrating good sensitivity and excellent specificity of IHC when used with appropriate protocols for ALK detection.

All the data required to estimate confidence intervals (CIs) are included in our paper. Using an online tool to estimate $\mathrm{CIs}^{6}$ based on the data in Table $3^{1}$ if any IHC staining is considered positive $(1,2$, or $3+$ intensity, and using 0.001 as a substitute for 0 where required), the D5F3 antibody (and the ALK1 clone) had a sensitivity of $100 \%$ (95\% CI $64.6-100 \%)$, specificity $99.0 \% \quad(95 \%$ CI $\quad 97.8-$ $99.5 \%)$, negative predictive value $100 \%$ (95\% CI 99.3-100\%), and positive predictive value 53.8\% (95\% CI 29.1-76.8\%). However, as shown in Table 3, most true positive cases stained only weakly with the ALK1 clone (5 of 7 with $1+$ intensity) compared to the D5F3 clone where most cases stained more strongly (only 2 of 7 with weak $1+$ staining). $5 \mathrm{~A} 4$ had a sensitivity of $100 \%$ (95\% CI $64.6-100 \%$ ), specificity of $98.1 \% \quad(95 \%$ CI $\quad 96.7-99.0 \%$ ), a negative predictive value of $100 \%$ (95\% CI 99.3$100 \%)$, and positive predictive value of $38.9 \%$ (95\% CI $20.3-61.4 \%$ ). While this shows that the $95 \%$ CIs are relatively wide as a result of the low numbers of ALK FISH-positive cases, the very high negative predictive value of IHC (100\% with 95\% CI 99.3$100 \%$ ) supports our conclusion that ALK IHC is a valuable screening method where false negatives are important to avoid. Screening strategies require confirmation by a more accurate testing method and the relatively low positive predictive values support confirmation by FISH testing as outlined in our paper. ${ }^{1}$

The optimal diagnostic algorithm to detect ALK in clinical samples is still evolving and will likely be influenced not only by test sensitivity and specificity but also by local expertise and cost effectiveness. Our study and others support the inclusion of IHC within this algorithm. The recently published consensus Molecular Testing Guidelines from CAP/IASLC/ $\mathrm{AMP}^{7}$ recommend that 'ALK immunohistochemistry, if carefully validated, may be considered as a screening methodology to select specimens for $A L K$ FISH testing.' In addition, the IASLC Atlas of ALK Testing in Lung Cancer ${ }^{5}$ states that 'a high diagnostic specificity and sensitivity for ALK IHC' compared to FISH has been confirmed in many studies.' As in our paper, the authors of the ALK Atlas recommend that ALK FISH is used to verify all IHC-positive cases until further data are obtained regarding clinicopathological associations of ALK IHC-positive cases and importantly response to ALK inhibition. ${ }^{5}$ Finally, we are in complete agreement that further prospective studies are required to establish the optimal diagnostic strategy for detection of $A L K$ gene rearrangements in NSCLC. 